\title{
Commentary - Marijuana use during pregnancy and premature birth: A problem likely to worsen
}

\author{
Joseph J. Volpe, M.D. ${ }^{\mathrm{a}, \mathrm{b}, *}$ \\ ${ }^{a}$ Department of Neurology, Harvard Medical School, Boston, MA, USA \\ ${ }^{\mathrm{b}}$ Department of Pediatric Newborn Medicine, Harvard Medical School, Boston, MA, USA
}

Received 30 December 2019

Accepted 30 December 2019

Keywords: Marijuana, pregnancy, prematurity, lactation, brain disturbance

\section{Introduction}

This Commentary was provoked by three recent reports, concerning (1) the association between marijuana (cannabis) use during pregnancy and perinatal outcomes [1], (2) the increasing use of cannabis during pregnancy (see later), and (3) recent insights into the effects of cannabis on the developing brain [2]. Taken together, the data suggest that prenatal cannabis use has the potential to harm the developing brain and support the recent opinion of the Committee on Obstetric Practice of the American College of Obstetrics and Gynecology to discourage use of marijuana during pregnancy and lactation [3]. Notably, the opinion is consistent with the data to follow and with the well-established facts that tetrahydrocannabinol (THC), the principal psychoactive component of marijuana, crosses the placental barrier readily and during lactation can be identified in breast milk $[2,4,5]$.

\footnotetext{
*Address for correspondence: Joseph J. Volpe, M.D., Department of Pediatric Newborn Medicine, Brigham and Women's Hospital, 221 Longwood Avenue, Room 343C, Boston, MA 02115, USA. Tel.: +1 617525 4145. E-mail: Joseph.volpe@childrens. harvard.edu.
}

\section{Recent clinical developments provoking concern}

Recent clinical observations indicate that cannabis use is significantly associated with an increased risk of preterm birth. Previous studies have been marred by small numbers, faulty study design and lack of careful attention to confounders. A recent study of 661,617 pregnant women in Canada appears to avoid these concerns [1]. The principal finding was a 2-fold higher rate of preterm birth less than 37 weeks' gestation among cannabis users during pregnancy $(12.0 \%$ in cannabis users; $6.1 \%$ in nonusers). The relative risk increased to 3-fold for births at less than 32 weeks' gestation. With rigorous control of confounders relative risks were slightly less but significant. Thus, reported cannabis use was significantly associated with an increased risk of preterm birth. (Statistically significantly higher rates of other adverse outcomes (small for gestational age, placental abruption, transfer to a neonatal intensive care unit and 5-minute Apgar scores less than 4) were also found but were less robust than the effect on premature birth.)) 
A second recent development of concern is that the use of cannabis during pregnancy has been increasing in recent years. In a particularly large recent study $(n=4000)$ of cannabis use among pregnant women in 2002-2003 vs. those in 2016-2017, the prevalence of past month use increased from $3.4 \%$ to $7.0 \%$ overall [6]. Notably use in the first trimester increased from $5.7 \%$ to $12.1 \%$. The extent to which this increase relates to a perception that cannabis is harmless, to increasing availability through legalization, or to increased prescribing for treatment of morning sickness is unclear. Likely, all of these factors are relevant.

A third relatively recent development is an apparent increase in cannabis potency in the last several decades. In one study of 38,681 samples of DEA confiscated materials, the potency of illicit cannabis plant material rose from approximately 4\% in 1995 to approximately $12 \%$ in 2014 [7]. In a similar study the increase in potency was from $3.4 \%$ in 1993 to $8.8 \%$ in 2008 [8]. This factor is critical to consider when assessing studies of the risk of cannabis use during pregnancy performed in decades past, especially studies suggesting no adverse effects of cannabis use.

\section{Cannabinoids and brain development}

To consider any potential adverse effects of cannabis use on human brain development, we should consider first the major developmental events occurring in human brain during pregnancy and early postnatal period. These events are reviewed in detail elsewhere [9] and consist in approximate chronological order from the first trimester principally of progenitor cell development, cell fate determination, neuronal proliferation, neuronal migration, glial differentiation, oligodendroglial development (in preparation for myelination), axonal outgrowth, cortical neuronal differentiation, synaptogenesis and connectivity. Many of these events are influenced by endogenous ligands (endocannabinoids [EC]) acting on specific EC receptors) [2]. These receptors have been identified in developing human brain early in gestation [10, 11] in germinative zones and especially in mesocorticolimbic brain structures [2]. [Mesocorticolimbic structures especially include the meso-ventral tegmental area in midbrain, amygdala, hippocampus, striatum, and prefrontal cortex]. Although specific EC receptors are especially prominent in mesocorticolimbic structures, the broad connections of these structures can influence the development of neural circuits more extensively.
Because these EC receptors are expressed at glutamatergic, GABAergic and dopaminergic terminals, their activation can lead to a wide array of developmental effects, including those related to cellular differentiation and circuit formation of the forebrain [2].

The particular concentration of EC receptors in mesocorticolimbic structures is important as determinants of the structures' particular neurological functional roles. Thus, these areas are involved later in such neurological functions as emotional control, motivation, cognition, and executive functions. Notably, these functions are difficult to quantitate in infants and young children, and thus some previous studies that suggest no clear deficits in infants and children born to women who used cannabis during pregnancy should be interpreted cautiously. Although not all findings are consistent, the data generally show in infants and young children exposed in utero no clear effect on global IQ but deficits in executive functioning, including attentional behavior, verbal memory, abstract and visual reasoning $[4,12]$. Importantly, however, the cohorts were exposed in utero to marijuana likely lower in potency than in today's cannabis. Current data are needed.

The anatomical substrate of any deleterious neurodevelopmental effects related to cannabis exposure in utero remains to be defined clearly in human infants, but studies in experimental models provide insight and guidance. A particularly important feature has been abnormal development of the prefrontal cortex, a cognitive hub whose neurodevelopmental perturbation has been linked to long-term behavioral deficits [2]. Additionally, disturbances in hippocampal structures also are considered critical.

Because cannabinoids can appear in breast milk, breast feeding significantly prolongs the potential period of direct transfer of cannabinoids consumed by the mother to the infant. A crucial event in the first months of post-term life is activation of a developmental switch of GABAergic cortical neurons from excitatory to inhibitory [13]. In animal models [2] this switch is retarded by exposure to exogenous cannabinoids. Because this switch is critical for specification of cognitive critical periods, failure of its activation could adversely affect cognitive development.

\section{Cannabinoids and prematurity}

The finding of increased rate of prematurity after cannabis use during pregnancy (see earlier) raises the 
concerning possibility of a synergistic, deleterious effect on brain development. As discussed in detail elsewhere [9], premature infants exhibit a high likelihood of dysmaturity of both gray matter and white matter structures. The dysmaturity relates to a variety of insults related to premature birth and appears to be initiated by the widely prevalent cerebral white matter injury [9]. The maturational disturbances involve many of the structures expected to be vulnerable to cannabis exposure, including striatum, axonal tracts and cerebral cortical structures, especially limbic structures. Indeed, recent advanced MRI findings show that in the premature infant the prefrontal cortex and the hippocampus exhibit impaired development $[14,15]$. Other work has demonstrated abnormalities of striatum and thalamus [16]. Thus, there appears to be a confluence of key brain structures affected independently by cannabinoids and by premature birth. The recent finding of enhanced rates of prematurity in cannabis-exposed pregnancies (see earlier) thus is of particular importance.

\section{Conclusions}

Recent observations, as described above, suggest that cannabis of increasingly high potency is used increasingly during pregnancy and may lead to the concerning combination of premature birth and brain dysmaturation. The combination is especially disturbing because the neural targets of cannabis exposure and prematurity overlap considerably. Overall, these considerations strongly support the ACOG Committee opinion that "women who are pregnant or women contemplating pregnancy should be encouraged to discontinue marijuana use" [3]. Although more data concerning lactating women are needed, because cannabis appears in breast milk, marijuana use in women breastfeeding also should be "discouraged" [3].

\section{References}

[1] Corsi DJ, Walsh L, Weiss D, Hsu H, El-Chaar D, Hawken S, et al. Association between self-reported prenatal cannabis use and maternal, perinatal, and neonatal outcomes. JAMA. 2019;322:145-52.
[2] Hurd YL, Manzoni OJ, Pletnikov MV, F.S. L, S. B, M. M. Cannabis and the developing brain: Insigjhts into its longlasting effects. J Neurosci. 2019;39:8250-8.

[3] Committee on Obstetric Practice American College of Obstetricians and Gynecologists, Committee Opinion Number 684. Delayed umbilical cord clamping after birth. Obstet Gynecol. 2017;129:e5-e10.

[4] Huizink AC. Prenatal cannabis exposure and infant outcomes: Overview of studies. Prog Neuropsychopharmacol Biol Psychiatry. 2014;52:45-52.

[5] Baker T, Datta P, Rewers-Felkins K, Thompson H, Kallem RR, Hale TW. Transfer of inhaled cannabis into human breast milk. Obstet Gynecol. 2018;131:783-8.

[6] Volkow ND, Han B, Compton WM, McCance-Katz EF. Self-reported medical and nonmedical cannabis use among pregnant women in the United States. JAMA. 2019;322:167-9.

[7] ElSohly MA, Mehmedic Z, Foster S, Gon C, Chandra S, Church JC. Changes in cannabis potency over the last 2 decades (1995-2014): Analysis of current data in the United States. Biol Psychiatry. 2016;79:613-9.

[8] Mehmedic Z, Chandra S, Slade D, Denham H, Foster S, Patel AS, et al. Potency trends of Delta9-THC and other cannabinoids in confiscated cannabis preparations from 1993 to 2008. J Forensic Sci. 2010;55:1209-17.

[9] Volpe JJ. Dysmaturation of premature brain: Importance, cellular mechanisms and potential interventions. Pediatr Neurol. 2019;95:42-66.

[10] Biegon A, Kerman IA. Autoradiographic study of pre- and postnatal distribution of cannabinoid receptors in human brain. Neuroimage. 2001;14:1463-8.

[11] Mato S, Del Olmo E, Pazos A. Ontogenetic development of cannabinoid receptor expression and signal transduction functionality in the human brain. Eur $\mathrm{J}$ Neurosci. 2003; 17:1747-54.

[12] Fried PA, Smith AM. A literature review of the consequences of prenatal marihuana exposure. An emerging theme of a deficiency in aspects of executive function. Neurotoxicol Teratol. 2001;23:1-11.

[13] Abend NS, Jensen FE, Inder TE, Volpe JJ. Neonatal seizures. In: Volpe JJ, Inder TE, Darras BT, deVries LS, duPlessis AJ, Neil JJ, et al., editors. Volpe's Neurology of the Newborn. 6th ed. Philadelphia: Elsevier; 2018. p. 275-321.

[14] Thompson DK, Adamson C, Roberts G, Faggian N, Wood SJ, Warfield SK, et al. Hippocampal shape variations at term equivalent age in very preterm infants compared with term controls: Perinatal predictors and functional significance at age 7. Neuroimage. 2013;70:278-87.

[15] Bouyssi-Kobar M, Brossard-Racine M, Jacobs M, Murnick J, Chang T, Limperopoulos C. Regional microstructural organization of the cerebral cortex is affected by preterm birth. Neuroimage Clin. 2018;18:871-80.

[16] Kinney HC, Volpe JJ. Encephalopathy of prematurity: Neuropathology. In: Volpe JJ, Inder TE, Darras BT, deVries LS, duPlessis AJ, Neil JJ, et al., editors. Volpe's Neurology of the Newborn. Chapter 14, 6th ed. Philadelphia, PA: Elsevier; 2018. p. 389-404. 